\title{
Scattering and space-charge effects in Wigner Monte Carlo simulations of single and double barrier devices
}

\author{
Viktor Sverdlov • Tibor Grasser · Hans Kosina • \\ Siegfried Selberherr
}

Published online: 9 December 2006

(C) Springer Science + Business Media, LLC 2007

\begin{abstract}
Transport in single and double barrier devices is studied using a Monte Carlo solver for the Wigner transport equation. This approach allows the effects of tunneling and scattering to be included. Several numerical methods have been improved to render the Wigner Monte Carlo technique more robust, including a newly developed particle annihilation algorithm. A self-consistent iteration scheme with the Poisson equation was introduced. The role of scattering and space charge effects on the electrical characteristics of $n-i-n$ nanostructures, ultra-scaled double gate MOSFETs, and GaAs resonant tunneling diodes is demonstrated.
\end{abstract}

Keywords Device simulation · Quantum transport . Wigner equation $\cdot$ Monte Carlo method

\section{Introduction}

As the channel length is scaled down to $30 \mathrm{~nm}$, quantum effects such as direct source-to-drain tunneling start affect-

\author{
V. Sverdlov $(\varangle) \cdot$ T. Grasser $\cdot$ H. Kosina $\cdot$ S. Selberherr \\ Institute for Microelectronics, TU Vienna, Gusshausstr. 27-29, \\ A-1040 Vienna, Austria \\ e-mail: sverdlov@iue.tuwien.ac.at \\ T. Grasser \\ e-mail: grasser@iue.tuwien.ac.at \\ H. Kosina \\ e-mail: kosina@iue.tuwien.ac.at \\ S. Selberherr \\ e-mail: selberherr@iue.tuwien.ac.at
}

ing the device characteristics, and new simulation tools are needed to adequately describe transport. At the same time there are growing evidences that scattering still controls the current in decananometer devices. Recent studies demonstrate that even for devices with a channel length as short as $15 \mathrm{~nm}$ scattering will play a significant role [1] and therefore determine the current, in accordance with estimations of the mean-free path in MOSFET structures [2]. The crossover from diffusive to ballistic transport in Si nanowire transistors occurs at approximately $2 \mathrm{~nm}$ [3], a much shorter distance than previously anticipated. An adequate transport model for ultra-scaled MOSFETS must therefore account for quantum mechanical and dissipative effects simultaneously.

A numerical model based on the Wigner function formalism can handle both quantum effects and dissipation. It reduces to a semiclassical transport description in the contacts, providing an important advantage of a seamless treatment of the transition between classical and quantum-mechanical device regions [4]. Realistic scattering processes can be easily embedded into the Wigner equation via Boltzmann-like scattering integrals [5], which makes it attractive for realistic device simulations.

In this work we apply the Wigner function formalism to demonstrate the role of scattering and space charge effects on the electrical characteristics of single and double barrier devices. $N-i-n$ structures, double gate field-effect transistors (DG FET), and resonant tunneling diodes (RTD) are considered. Several numerical methods have been improved to render the Wigner MC technique more robust, including the separation of a classical force, discretization of the Wigner potential, and a particle annihilation algorithm. A self-consistent iteration scheme with the Poisson equation was introduced. 


\section{Wigner function method}

The kinetic equation for the Wigner function $f_{w}(\mathbf{r}, \mathbf{k}, t)$ is similar to the Boltzmann equation $[6, ?]$ :

$$
\begin{aligned}
& \left(\frac{\partial}{\partial t}+\mathbf{v} \cdot \nabla_{r}\right) f_{w} \\
& =\int V_{w}\left(\mathbf{r}, \mathbf{k}^{\prime}-\mathbf{k}\right) f_{w}\left(\mathbf{k}^{\prime}, \mathbf{r}, t\right) \mathrm{d} \mathbf{k}^{\prime}+\left(\frac{\partial f_{w}}{\partial t}\right)_{\text {coll }},
\end{aligned}
$$

The Wigner potential $V_{w}(\mathbf{r}, \mathbf{k})$ enters the nonlocal operator in the right-hand side. In case of slowly varying potentials the nonlocal operator reduces to the classical force term. Following [8], one can introduce a spectral decomposition of the potential profile $V(x)$ into a slowly varying, classical component and a rapidly varying component treated quantummechanically.

$V(\mathbf{r})=V_{\mathrm{cl}}(\mathbf{r})+V_{\mathrm{qm}}(\mathbf{r})$.

This separation of the potential into a classical and a quantum mechanical contribution improves the stability of the numerical solution method.

The Wigner function formalism treats scattering and quantum mechanical effects on equal footing by using the corresponding scattering integrals. By analogy to the Monte Carlo (MC) methods used for the Boltzmann transport equation, one would try to solve the Wigner transport Eq. (1) by means of the MC technique. The development of MC methods for the Wigner equation, however, is hampered by the fact that the integral kernel is no longer positively definite, as it is in the semi-classical case. This so-called negative sign problem will lead to exponentially growing variances of the Markov Chain MC method [7]. The Wigner potential operator can also be viewed as a generation term of positive and negative numerical particles [4]. In this picture the sign problem shows up in the avalanche of numerical particles generated. A stable MC method can be achieved by means of a particle annihilation algorithm. To solve the sign problem an operator splitting method was proposed in [9], where the operator is separated into a main part allowing for a straightforward MC solution and a perturbation. The result of the perturbation operator applied to the solution is stored in a discrete form on a phase space mesh and is used in an iteration scheme. Especially for larger meshes these iterations may become prohibitively time consuming. We have developed a different strategy and construct an operator, whose application on the solution gives a small residual. The algorithm attempts to minimize the residual without iterations. The minimization is achieved by construction: The Wigner potential operator generates two numerical particles with opposite weight, whereas the initial particle state persists. Out of this three particles two are stored on the annihilation mesh. The particles to be stored are chosen such that the weights in the related mesh cells are minimized. One may choose the weight of the particle continuing the trajectory to be of the same sign as the incoming one. This scheme conserves current exactly.

To resolve the negative parts of the Wigner function a certain fraction of negative trajectories has to be constructed.

\section{Results}

The method has been applied to double-gate MOSFETs. In the coherent mode, where scattering is neglected, a comparison to conventional Schrödinger solvers can be performed. In order to estimate the tunneling component of current the Wigner Monte Carlo simulations were carried out for a MOSFET with a gate length of $10 \mathrm{~nm}$. Potential profiles were calculated self-consistently for a drain-source voltage of $0.4 \mathrm{~V}$ and different gate biases using the device simulator MINIMOS-NT. Good agreement between the two approaches is observed, as displayed in Fig. 1. The difference between quantum ballistic and semiclassical simulations is due to the additional contribution from electrons tunneling through the potential barrier.

An important advantage of the Wigner Monte Carlo method is that it allows inclusion of dissipative scattering processes. Scattering is introduced via the Boltzmann scattering integrals specific to the system under investigation. The inclusion of scattering stabilizes the Wigner Monte Carlo

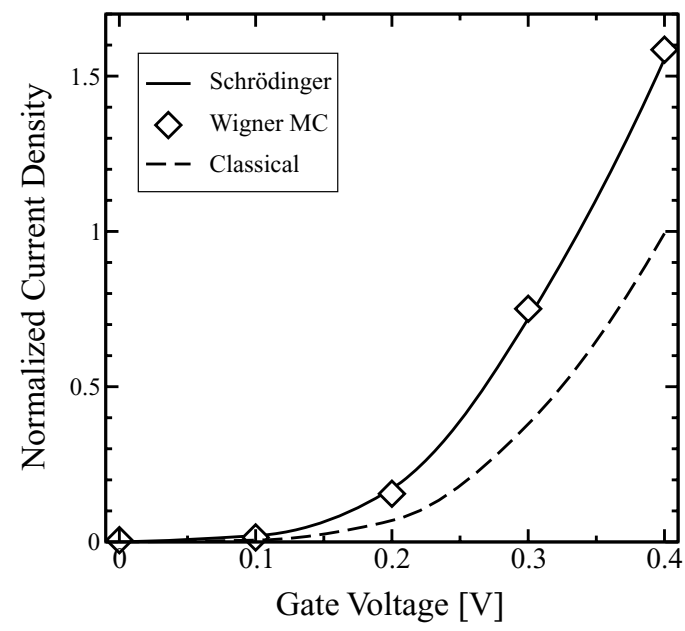

Fig. 1 Classical ballistic current density normalized to its value at $V_{G}=0.4 \mathrm{~V}$ and relative quantum mechanical current density obtained with the Wigner Monte Carlo (open symbols). Symbol size shows the statistical uncertainty for the Wigner Monte Carlo simulations. Additional source-to-drain tunneling current component is clearly visible. Current densities found from the solution of the Schrödinger equation with open boundary conditions are also shown 


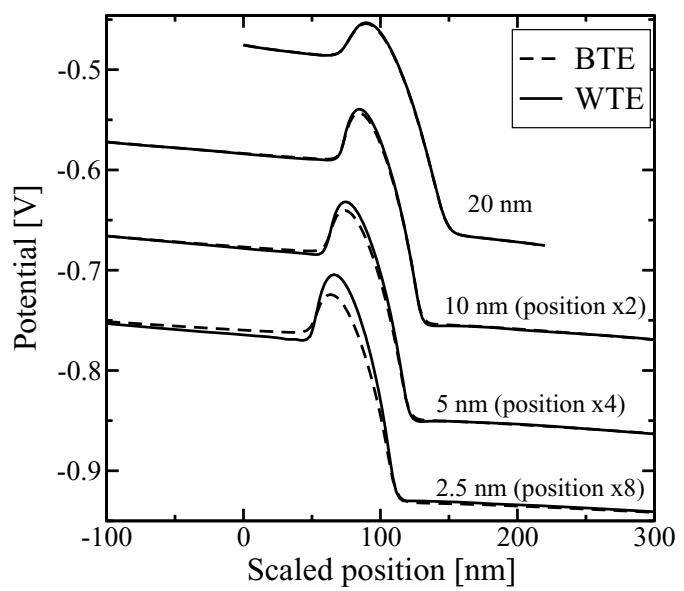

Fig. 2 Self-consistent potential profiles calculated for $n-i-n$ structure with Wigner (solid lines) and Boltzmann (dashed lines) transport equations. For long $n-i-n$ structures results are similar. For short $n-i-n$ structures additional charge due to tunneling electrons results in higher potential barrier

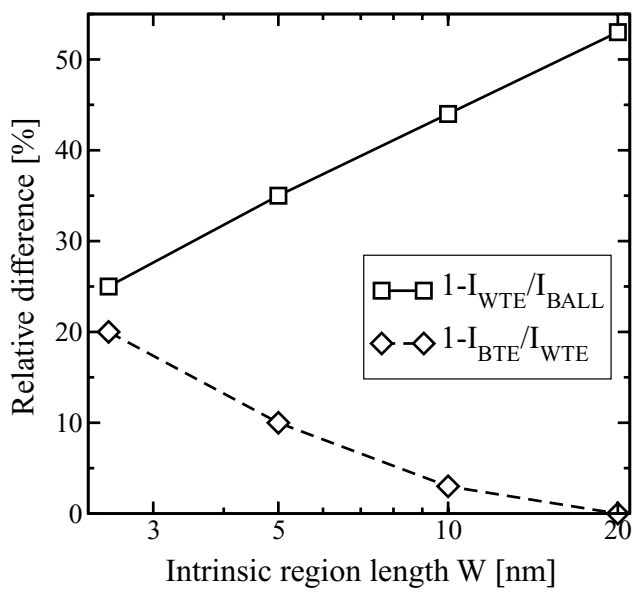

Fig. 3 Relative difference between currents calculated with the Wigner and Boltzmann Monte Carlo methods (diamonds) and calculated with the Wigner MC for an $n-i-n$ structure, with and without scattering in the intrinsic region

simulations. The carrier concentration can be used to update the potential in the device by solving the Poisson equation. A superimposed iteration loop makes the Wigner-Poisson solver self-consistent. An example of self-consistent potentials for Si $n-i-n$ structures with an intrinsic region of length $W$ ranging from $20 \mathrm{~nm}$ to $2.5 \mathrm{~nm}$, as calculated with Wigner and classical Monte Carlo, is shown in Fig. 2. The doping profile is assumed to increase gradually from the intrinsic channel to the highly doped contacts over the same distance $W$. Electron-phonon and Coulomb scattering were included. As expected, for thick $W$ the classical and quantum calculations yield similar results for the self-consistent potential. For $W=2.5 \mathrm{~nm}$ an extra space charge due to electrons tunneling under the barrier becomes important, rising the potential

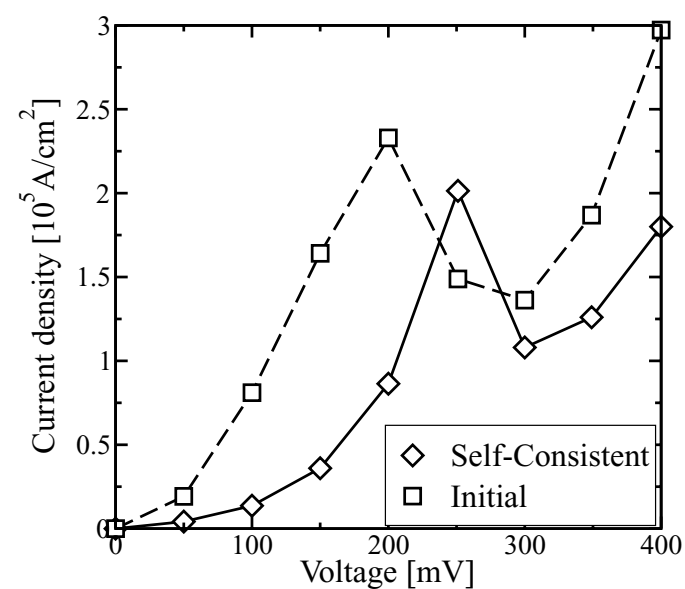

Fig. 4 Typical $I V$ curve of RTD, calculated self-consistently (solid line), contrasted against a non self-consistent characteristics

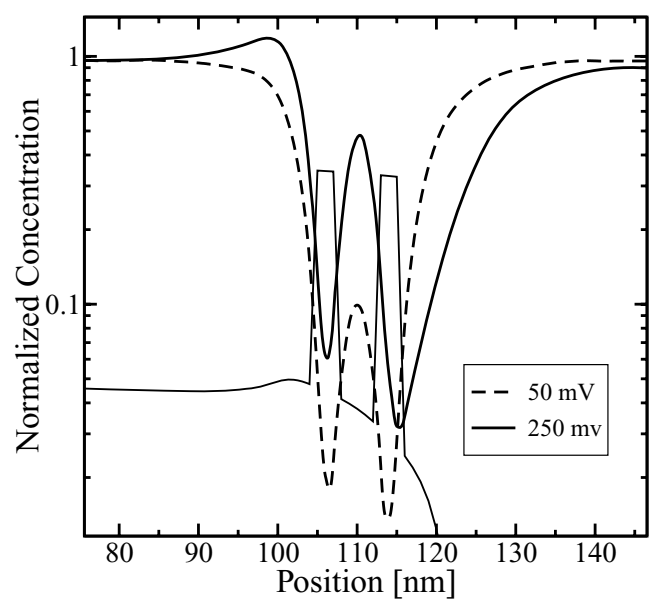

Fig. 5 Normalized electron concentration off-resonance (dashed line) and at resonance (solid line) in RTD

barrier. However, the current found with the Wigner function method is approximately $20 \%$ higher compared to its classical value determined from a self-consistent solution of the Boltzmann and the Poisson equations.

Relative differences between $I_{W I G}$ and the current $I_{B A L L}$ computed for a "ballistic" device with scattering inside the intrinsic and transition regions turned off is shown in Fig. 3. For $\mathrm{W}=2.5 \mathrm{~nm}$ the relative differences in current due to quantum effects and scattering in the barrier are still of the order of $25 \%$ and cannot be neglected.

The Wigner function method gives accurate results not only for single-barrier devices, but can also be applied to purely quantum-mechanical systems such as resonant tunneling diodes [4]. A typical output characteristic of a GaAs resonant tunneling diode, with and without space-charge effects taken into account, is shown in Fig. 4. Scattering with polar optical phonons as well as Coulomb scattering in the 
contacts doped to $10^{18} \mathrm{~cm}^{-3}$ is considered. A region of negative differential resistance common to transport via a resonant level is clearly visible after the resonance peak at $250 \mathrm{mV}$ applied voltage. A self-consistent solution of the Wigner and Poisson equation is mandatory for the correct determination of the resonance position. Before the barrier, an accumulation layer forms, depending on the applied voltage, as seen in Fig. 5. This results in a voltage shift of the resonance peak of the $I / V$ characteristics shown in Fig. 4. A typical distribution of the concentration in resonance condition and off-resonance is presented in Fig. 5. The amount of charge localized in the potential well is much higher at resonance. It leads to a potential barrier increase and also contributes to the shift of the resonant peak.

\section{Conclusions}

The Wigner function method is shown to be a comprehensive tool to address simulation needs of emerging nanoelectronic devices. It treats tunneling and dissipative effects on equal footing, allowing for inclusion of realistic scattering mechanisms. The method guarantees a seamless link between the quantum region and classical contact regions making it attractive for device simulations. A Monte Carlo algorithm for solving the Wigner equation with non-positive kernel is developed. The method is applied to single and double barrier nanostructures. It demonstrates the importance of both quantum-mechanical and scattering effects in emerging nanodevices.

Acknowledgments We gratefully acknowledge financial support from the Austrian Science Fund FWF, project P17285-N02, and the European Commission, project SINANO IST-506844.

\section{References}

1. Palestri, P. et al.: Understanding quasi-ballistic transport in nanoMOSFETs: Part I-Scattering in the channel, and in the drain. IEEE Trans. Electron Devices 52(12), 2727 (2005)

2. Jungemann, C.: et al.: Investigation of strained $\mathrm{Si} / \mathrm{SiGe}$ devices by MC simulation, Solid-State Electron. 48(8), 1417 (2004)

3. Gilbert, M. et al.: Phonon-assisted ballistic to diffusive crossover in silicon nanowire transistors. J. Appl. Phys. 98(9), 094303 (2005)

4. Kosina, H. et al.: A Monte Carlo method seamlessly linking quantum and classical transport calculations. J. Computational Electronics 2(2-4), 147 (2002)

5. Frensley, W.: Boundary conditions for open quantum systems driven far from equilibrium. Rev. Modern Phys. 62(3), 745 (1990)

6. Wigner, E.: On the quantum correction for thermodynamic equilibrium. Physi. Revi. 40, 749 (1932)

7. Kosina, H., Nedjalkov, M.: Handbook of theoretical and computational nanotechnology In: 10 chapter Wigner Function Based Device Modeling, pp. 731-763. American Scientific Publishers Los Angeles (2006), (in print)

8. Gehring, A., Kosina, H.: Wigner-function based simulation of quantum transport in scaled dg-mosfets using the monte carlo method. J. Computational Electronics 4(1-2), 67 (2005)

9. Nedjalkov, M. et al.: Operator-split method for variance reduction in stochastic solutions for the wigner equation. Monte Carlo Methods Appl. 10(3-4), 461 (2004) 